\title{
Optimal Homotopy Asymptotic Method for Solving the Linear Fredholm Integral Equations of the First Kind
}

\author{
Mohammad Almousa and Ahmad Ismail \\ School of Mathematical Sciences, Universiti Sains Malaysia (USM), 11800 Gelugor, Penang, Malaysia \\ Correspondence should be addressed to Mohammad Almousa; mohammadalmousa12@yahoo.com
}

Received 20 April 2013; Accepted 16 June 2013

Academic Editor: Santanu Saha Ray

Copyright ( 2013 M. Almousa and A. Ismail. This is an open access article distributed under the Creative Commons Attribution License, which permits unrestricted use, distribution, and reproduction in any medium, provided the original work is properly cited.

The aim of this study is to present the use of a semi analytical method called the optimal homotopy asymptotic method (OHAM) for solving the linear Fredholm integral equations of the first kind. Three examples are discussed to show the ability of the method to solve the linear Fredholm integral equations of the first kind. The results indicated that the method is very effective and simple.

\section{Introduction}

Integral equations of the first kind arise in several applications. These include applications in biology, chemistry, physics, and engineering. In recent years, much work has been carried out by researchers in mathematics and engineering in applying and analyzing novel numerical and semi analytical methods for obtaining solutions of integral equations of the first kind. Among these are the homotopy analysis method [1], operational Tau method [2], homotopy perturbation method [3], Adomian decomposition [3], quadrature rule [4], and automatic augmented Galerkin algorithms [5].

In this study, we develop the optimal homotopy asymptotic method (OHAM), which was proposed by Marinca et al. $[6,7]$, for solving the linear Fredholm integral equations of the first kind. This method is characterized by it is convergence criteria which are more flexible than other methods.

The general form of the linear Fredholm integral equations of the first kind is

$$
f(s)=\int_{a}^{b} K(s, t) g(t) d t,
$$

where $a$ and $b$ are constant and the functions $k(s, t)$ and $f(s)$ are known.

It should be noted that OHAM has been applied to the nonlinear Fredholm integral equations of the second kind by [8].

\section{Application of OHAM to the Linear Fredholm Integral Equations of the First Kind}

In this section, we formulate the optimal homotopy asymptotic method (OHAM) for solving the linear Fredholm integral equations of the first kind following the procedure as outlined in $[6,7]$ and other papers. Let us consider a form of the linear Fredholm integral equation of the first kind:

$$
f(s)-\int_{a}^{b} K(x, t) g(t) d t=0 .
$$
lows:

Using OHAM, we can obtain a family of equations as fol-

$$
\begin{aligned}
&(1-p) {[L(g(s, p))+f(s)] } \\
&=H(p)[L(g(s, p))+f(s)+N(g(s, p))],
\end{aligned}
$$

where $p \in[0,1]$ is an embedding parameter, $g(s, p)$ is unknown function, and $H(p)$ is an (nonzero) auxiliary function for $p \neq 0$ and $H(0)=0$ and given as $H(p)=\sum_{j=1}^{m} c_{j} p^{j}$ where $c_{j}, j=1,2, \ldots$, are auxiliary constants, and when $p=0$ and $p=1$ it holds that

$$
g(s, 0)=g_{0}(s), \quad g(s, 1)=g(s),
$$


respectively. For obtaining the approximate solution, we use Taylor's series expansion about $p$ as follows:

$$
g\left(s, p, c_{j}\right)=g_{0}(s)+\sum_{m=1}^{\infty} g_{m}\left(s, c_{j}\right) p^{m}, \quad j=1,2, \ldots
$$

If the series (5) convergence occurs when $p=1$, one has

$$
g\left(s, 1, c_{j}\right)=g_{0}(s)+\sum_{m=1}^{\infty} g_{m}\left(s, c_{j}\right), \quad j=1,2, \ldots
$$

Substituting (5) in (3) and equating the coefficients of like powers of $p$, we get as follows:

$$
\begin{gathered}
O\left(p^{0}\right): g_{0}(s)=-f(s), \\
O\left(p^{1}\right): g_{1}(s)=-c_{1} \int_{a}^{b} K(s, t) g_{0}(t) d t \\
O\left(p^{2}\right): g_{2}(s)=\left(1+c_{1}\right) g_{1}(s)-c_{1} \int_{a}^{b} K(s, t) g_{1}(t) d t \\
-c_{2} \int_{a}^{b} K(s, t) g_{0}(t) d t \\
O\left(p^{i}\right): g_{i}(s)=\left(1+c_{1}\right) g_{i-1}(s)+\sum_{j=2}^{i-1} c_{j} g_{i-j}(s) \\
-\sum_{k=1}^{i} c_{k} \int_{a}^{b} K(s, t) g_{i-k}(t) d t .
\end{gathered}
$$

For finding the constants $c_{1}, c_{2}, c_{3}, \ldots$, we can get the result of the $m$ th-order approximations as follows:

$$
g^{m}\left(s, c_{j}\right)=g_{0}(s)+\sum_{k=1}^{m} g_{k}\left(s, c_{j}\right), \quad j=1,2, \ldots, m .
$$

If we substitute (8) into (1) we obtain the residual equation

$$
R\left(s, c_{j}\right)=L\left(g^{m}\left(s, c_{j}\right)\right)+f(s)-\int_{a}^{b} K(s, t) g^{m}\left(t, c_{j}\right) d t .
$$

If $R\left(s, c_{j}\right)=0$, then $g^{m}\left(s, c_{j}\right)$ will be the exact solution. The least squares method can be used to determine $c_{1}, c_{2}, c_{3}, \ldots$ At first we consider the functional

$$
J\left(c_{j}\right)=\int_{a}^{b} R^{2}\left(s, c_{j}\right) d s .
$$

By using Galerkin's method we get the following system:

$$
\frac{\partial J}{\partial c_{j}}=2 \int_{a}^{b} R\left(s, c_{j}\right) \frac{\partial R}{\partial c_{j}} d s
$$

and then minimizing it to obtain the values of $c_{1}, c_{2}, \ldots, m$, we have

$$
\frac{\partial J}{\partial c_{1}}=\frac{\partial J}{\partial c_{2}}=\cdots=\frac{\partial J}{\partial c_{m}}=0 .
$$

With these constants, the approximate solution is determined.

\section{Numerical Examples and Discussion}

In this section, three examples of the linear Fredholm integral equations of the first kind were solved to show the efficiency of the present method. Maple software with long format and double accuracy was used to carry out the computations.

Example 1. We consider the following equation [9]:

$$
\frac{1}{2} \sin (s)=\int_{0}^{\pi / 2} \frac{2}{\pi} \sin (s) \sin (t) g(t) d t,
$$

for which the exact solution is $g(s)=\sin (s)$. Applying OHAM to the linear Fredholm integral equation of first kind yields

$$
\begin{gathered}
L(g(s, p))=g(s), \\
N(g(s, p))=-\int_{0}^{\pi / 2} \frac{2}{\pi} \sin (s) \sin (t) g(t) d t, \\
f(s)=\frac{1}{2} \sin (s)
\end{gathered}
$$

which satisfies

$$
\begin{gathered}
(1-p)\left[\left(g_{0}(s)+p g_{1}(s)+p^{2} g_{2}(s)+\cdots\right)+\frac{1}{2} \sin (s)\right] \\
=\left(p c_{1}+p^{2} c_{2}+p^{3} c_{3}+\cdots\right) \\
\times\left[\left(g_{0}(s)+p g_{1}(s)+p^{2} g_{2}(s)+\cdots\right)+\frac{1}{2} \sin (s)\right. \\
\quad-\int_{0}^{\pi / 2} \frac{2}{\pi} \sin (s) \sin (t)\left(g_{0}(t)+p g_{1}(t)\right. \\
\left.\left.+p^{2} g_{2}(t)+\cdots\right) d t\right] .
\end{gathered}
$$

Now we use (7) to obtain a series of problems:

$$
\begin{gathered}
O\left(p^{0}\right): g_{0}(s)=-\frac{1}{2} \sin (s) \\
O\left(p^{1}\right): g_{1}(s)=-c_{1} \int_{0}^{\pi / 2} \frac{2}{\pi} \sin (s) \sin (t) g_{0}(t) d t \\
O\left(p^{2}\right): g_{2}(s)=\left(1+c_{1}\right) g_{1}(s) \\
-c_{1} \int_{0}^{\pi / 2} \frac{2}{\pi} \sin (s) \sin (t) g_{1}(t) d t \\
-c_{2} \int_{0}^{\pi / 2} \frac{2}{\pi} \sin (s) \sin (t) g_{0}(t) d t
\end{gathered}
$$

Hence the solutions are

$$
\begin{gathered}
O\left(p^{0}\right): g_{0}(s)=-\frac{1}{2} \sin (s), \\
O\left(p^{1}\right): g_{1}(s)=\frac{1}{4} c_{1} \sin (s), \\
O\left(p^{2}\right): g_{2}(s)=\frac{1}{4}\left(1+c_{1}\right) c_{1} \sin (s) \\
-\frac{1}{8} c_{1}^{2} \sin (s)+\frac{1}{4} c_{2} \sin (s) .
\end{gathered}
$$


By substituting $g_{0}(s), g_{1}(s)$, and $g_{3}(s)$ solutions in (6), we obtain

$$
\begin{aligned}
g(s)= & -\frac{1}{2} \sin (s)+\frac{1}{4} c_{1} \sin (s) \\
& +\frac{1}{4}\left(1+c_{1}\right) c_{1} \sin (s) \\
& -\frac{1}{8} c_{1}^{2} \sin (s)+\frac{1}{4} c_{2} \sin (s) .
\end{aligned}
$$

For the calculations of the constants $c_{1}$ and $c_{2}$, the use of the technique mentioned in (8)-(12) yields

$$
c_{1}=6.000000004, \quad c_{2}=-24.00000002 .
$$

Substituting values in (18), the final solution becomes

$$
g(s)=\sin (s) .
$$

This is the exact solution.

Table 1 shows some numerical results of these solutions calculated according to the present method.

The exact solution, OHAM solution and absolute error of this example are shown in Figure 1.

Example 2. We consider the following equation [10]:

$$
\frac{1}{4} s^{2}=\int_{0}^{1} \frac{5}{2} s^{2} t^{2} g(t) d t
$$

for which the exact solution is $g(s)=(1 / 2) s^{2}$. Applying OHAM to the linear Fredholm integral equation of first kind yields

$$
\begin{gathered}
L(g(s, p))=g(s), \\
N(g(s, p))=-\int_{0}^{1} \frac{5}{2} s^{2} t^{2} g(t) d t, \\
f(s)=\frac{1}{4} s^{2}
\end{gathered}
$$

which satisfies

$$
\begin{aligned}
(1-p) & {\left[\left(g_{0}(s)+p g_{1}(s)+p^{2} g_{2}(s)+\cdots\right)+\frac{1}{4} s^{2}\right] } \\
= & \left(p c_{1}+p^{2} c_{2}+p^{3} c_{3}+\cdots\right) \\
\times & {\left[\left(g_{0}(s)+p g_{1}(s)+p^{2} g_{2}(s)+\cdots\right)+\frac{1}{4} s^{2}\right.} \\
& \left.\quad-\int_{0}^{1} \frac{5}{2} s^{2} t^{2}\left(g_{0}(t)+p g_{1}(t)+p^{2} g_{2}(t)+\cdots\right) d t\right] .
\end{aligned}
$$

TABLE 1: Numerical results of Example 1.

\begin{tabular}{lccc}
\hline$s$ & $g_{\text {exact }}$ & $g_{\text {OHAM }}$ & $\left|g_{\text {exact }}-g_{\text {OHAM }}\right|$ \\
\hline 0 & 0 & 0 & 0 \\
0.1 & 0.09983341665 & 0.09983341665 & 0 \\
0.2 & 0.1986693308 & 0.1986693308 & 0 \\
0.3 & 0.2955202067 & 0.2955202067 & 0 \\
0.4 & 0.3894183423 & 0.3894183423 & 0 \\
0.5 & 0.4794255386 & 0.4794255386 & 0 \\
0.6 & 0.5646424734 & 0.5646424734 & 0 \\
0.7 & 0.6442176872 & 0.6442176872 & 0 \\
0.8 & 0.7173560909 & 0.7173560909 & 0 \\
0.9 & 0.7833269096 & 0.7833269096 & 0 \\
1.0 & 0.8414709848 & 0.8414709848 & 0 \\
\hline
\end{tabular}

Now we use (7) to obtain a series of problems:

$$
\begin{gathered}
O\left(p^{0}\right): g_{0}(s)=-\frac{1}{4} s^{2}, \\
O\left(p^{1}\right): g_{1}(s)=-c_{1} \int_{0}^{1} \frac{5}{2} s^{2} t^{2} g_{0}(t) d t, \\
O\left(p^{2}\right): g_{2}(s)=\left(1+c_{1}\right) g_{1}(s) \\
-c_{1} \int_{0}^{1} \frac{5}{2} s^{2} t^{2} g_{1}(t) d t-c_{2} \int_{0}^{1} \frac{5}{2} s^{2} t^{2} g_{0}(t) d t .
\end{gathered}
$$

Hence the solutions are

$$
\begin{gathered}
O\left(p^{0}\right): g_{0}(s)=-\frac{1}{4} s^{2}, \\
O\left(p^{1}\right): g_{1}(s)=\frac{1}{8} c_{1} s^{2}, \\
O\left(p^{2}\right): g_{2}(s)=\frac{1}{8}\left(1+c_{1}\right) c_{1} s^{2}-\frac{1}{16} c_{1}^{2} s^{2}+\frac{1}{8} c_{2} s^{2} .
\end{gathered}
$$

By substituting $g_{0}(s), g_{1}(s)$, and $g_{3}(s)$ solutions in (6), we obtain

$$
g(s)=-\frac{1}{4} s^{2}+\frac{1}{8} c_{1} s^{2}+\frac{1}{8}\left(1+c_{1}\right) c_{1} s^{2}-\frac{1}{16} c_{1}^{2} s^{2}+\frac{1}{8} c_{2} s^{2} .
$$

For the calculations of the constants $c_{1}$ and $c_{2}$, the use of the technique mentioned in (8)-(12) yields

$$
c_{1}=6, \quad c_{2}=-24 \text {. }
$$

Substituting values in (26), the final solution becomes

$$
g(s)=\frac{1}{2} s^{2} .
$$

This is the exact solution.

Table 2 shows some numerical results of these solutions calculated according to the present method.

The exact solution, OHAM solution and absolute error of this example are shown in Figure 2. 


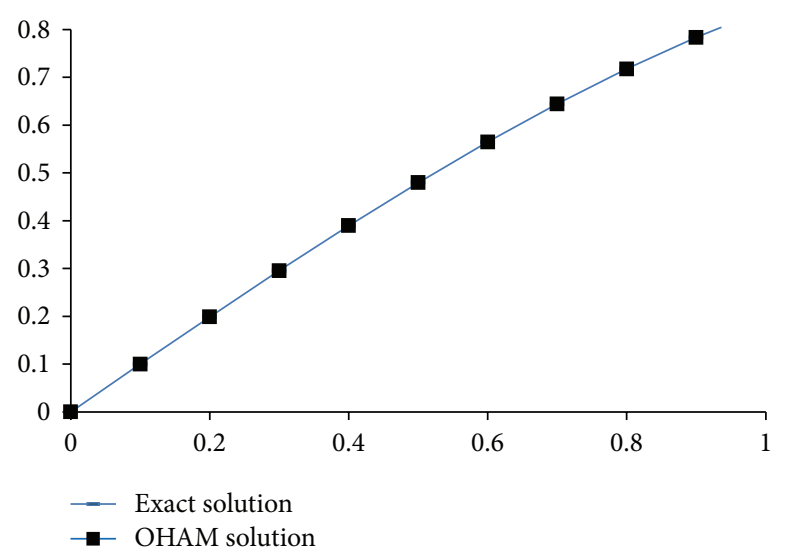

(a) Results for Example 1

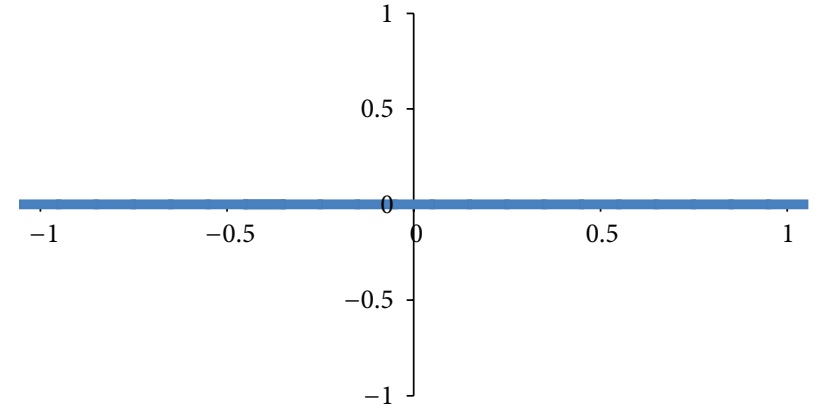

-- Absolute error for Example 1

Figure 1

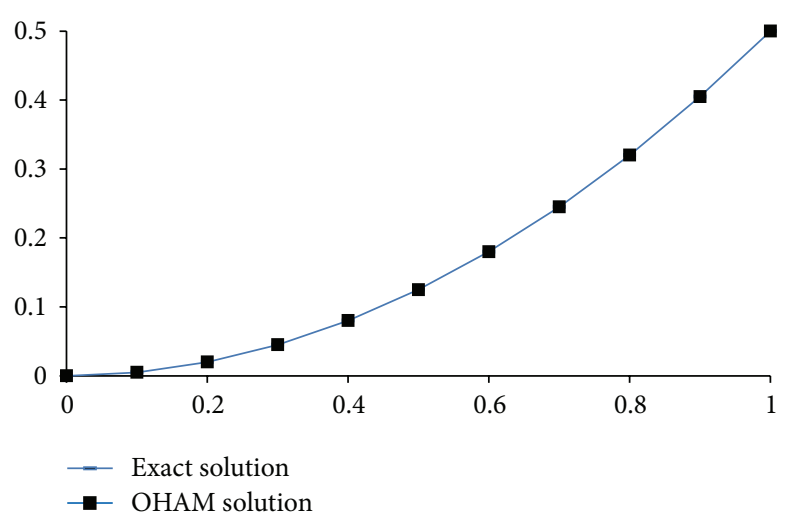

(a) Results for Example 2

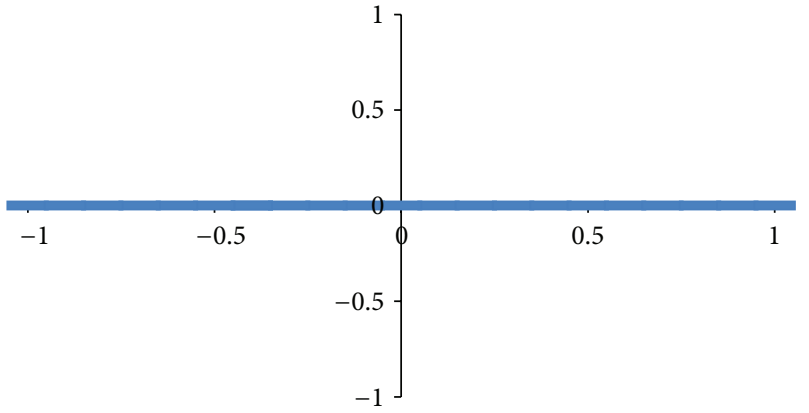

Absolute error for Example 2

(b) Absolute error for Example 2

Figure 2

TABLE 2: Numerical results of Example 2.

\begin{tabular}{lccc}
\hline$s$ & $g_{\text {exact }}$ & $g_{\text {OHAM }}$ & $\left|g_{\text {exact }}-g_{\text {OHAM }}\right|$ \\
\hline 0 & 0 & 0 & 0 \\
0.1 & 0.005 & 0.005 & 0 \\
0.2 & 0.02 & 0.02 & 0 \\
0.3 & 0.045 & 0.045 & 0 \\
0.4 & 0.08 & 0.08 & 0 \\
0.5 & 0.125 & 0.125 & 0 \\
0.6 & 0.18 & 0.18 & 0 \\
0.7 & 0.245 & 0.245 & 0 \\
0.8 & 0.32 & 0.32 & 0 \\
0.9 & 0.405 & 0.405 & 0 \\
1.0 & 0.5 & 0.5 & 0 \\
\hline
\end{tabular}

Example 3. We consider the following equation [9]:

$$
\frac{1}{2} s^{2}=\int_{0}^{1} 2 s^{2} t g(t) d t
$$

for which the exact solution is $g(s)=(1 / 2) s^{2}$. Applying $\mathrm{OHAM}$ to the linear Fredholm integral equation of first kind yields

$$
\begin{gathered}
L(g(s, p))=g(s), \\
N(g(s, p))=-\int_{0}^{1} 2 s^{2} t g(t) d t \\
f(s)=\frac{1}{2} s^{2}
\end{gathered}
$$

which satisfies

$$
\begin{aligned}
& (1-p)\left[\left(g_{0}(s)+p g_{1}(s)+p^{2} g_{2}(s)+\cdots\right)+\frac{1}{2} s^{2}\right] \\
& =\left(p c_{1}+p^{2} c_{2}+p^{3} c_{3}+\cdots\right) \\
& \quad \times\left[\left(g_{0}(s)+p g_{1}(s)+p^{2} g_{2}(s)+\cdots\right)+\frac{1}{2} s^{2}\right. \\
& \left.\quad-\int_{0}^{1} 2 s^{2} t\left(g_{0}(t)+p g_{1}(t)+p^{2} g_{2}(t)+\cdots\right) d t\right] .
\end{aligned}
$$




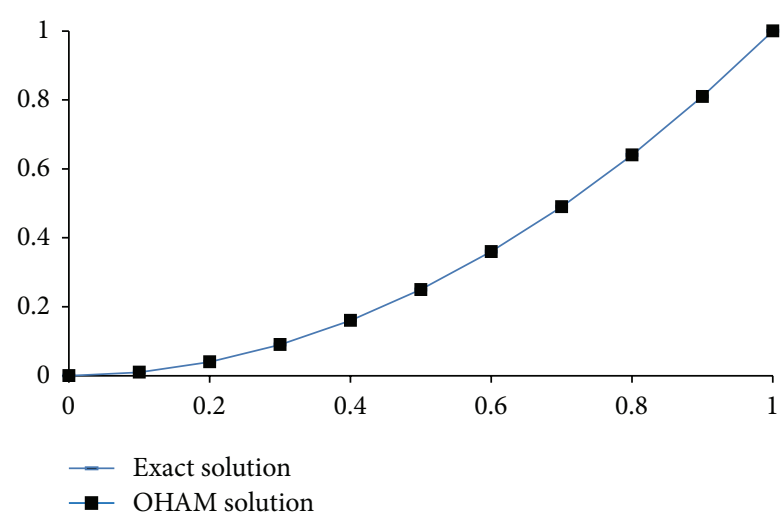

(a) Results for Example 3

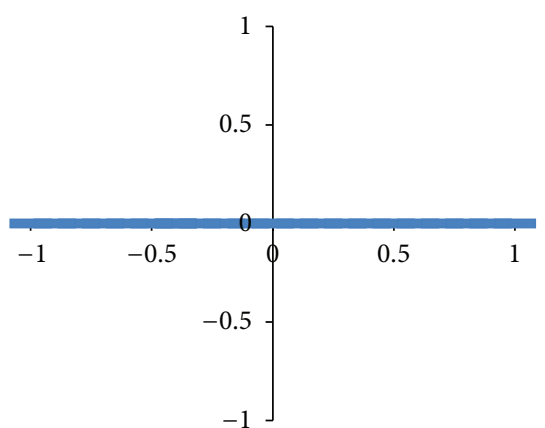

_-_ Absolute error for Example 3

(b) Absolute error for Example 3

Figure 3

Now we use (7) to obtain a series of problems:

$$
\begin{gathered}
O\left(p^{0}\right): g_{0}(s)=-\frac{1}{2} s^{2} \\
O\left(p^{1}\right): g_{1}(s)=-c_{1} \int_{0}^{1} 2 s^{2} t g_{0}(t) d t \\
O\left(p^{2}\right): g_{2}(s) \\
=\left(1+c_{1}\right) g_{1}(s)-c_{1} \int_{0}^{1} 2 s^{2} \operatorname{tg}_{1}(t) d t \\
-c_{2} \int_{0}^{1} 2 s^{2} \operatorname{tg}_{0}(t) d t .
\end{gathered}
$$

Hence the solutions are

$$
\begin{gathered}
O\left(p^{0}\right): g_{0}(s)=-\frac{1}{2} s^{2}, \\
O\left(p^{1}\right): g_{1}(s)=\frac{1}{4} c_{1} s^{2} \\
O\left(p^{2}\right): g_{2}(s)=\frac{1}{4}\left(1+c_{1}\right) c_{1} s^{2}-\frac{1}{8} c_{1}^{2} s^{2}+\frac{1}{4} c_{2} s^{2} .
\end{gathered}
$$

By substituting $g_{0}(s), g_{1}(s)$, and $g_{3}(s)$ solutions in (6), we obtain

$$
\begin{aligned}
g(s)= & -\frac{1}{2} s^{2}+\frac{1}{4} c_{1} s^{2}+\frac{1}{4}\left(1+c_{1}\right) c_{1} s^{2} \\
& -\frac{1}{8} c_{1}^{2} s^{2}+\frac{1}{4} c_{2} s^{2} .
\end{aligned}
$$

For the calculations of the constants $c_{1}$ and $c_{2}$, the use of the technique mentioned in (8)-(12) yields

$$
c_{1}=6, \quad c_{2}=-24 \text {. }
$$

Substituting values in (34), the final solution becomes

$$
g(s)=s^{2} .
$$

This is the exact solution.
TABLE 3: Numerical results of Example 3.

\begin{tabular}{lccc}
\hline$s$ & $g_{\text {exact }}$ & $g_{\text {OHAM }}$ & $\left|g_{\text {exact }}-g_{\text {OHAM }}\right|$ \\
\hline 0 & 0 & 0 & 0 \\
0.1 & 0.01 & 0.01 & 0 \\
0.2 & 0.04 & 0.04 & 0 \\
0.3 & 0.09 & 0.09 & 0 \\
0.4 & 0.16 & 0.16 & 0 \\
0.5 & 0.25 & 0.25 & 0 \\
0.6 & 0.36 & 0.36 & 0 \\
0.7 & 0.49 & 0.49 & 0 \\
0.8 & 0.64 & 0.64 & 0 \\
0.9 & 0.81 & 0.81 & 0 \\
1.0 & 1.0 & 1.0 & 0 \\
\hline
\end{tabular}

Table 3 shows some numerical results of these solutions calculated according to the present method.

The exact solution, OHAM solution and absolute error of this example are shown in Figure 3.

\section{Conclusions}

In this paper, we presented the application of the OHAM in solving the linear Fredholm integral equations of the first kind. This method was tested on three different examples. This method proved to be an accurate and efficient technique for finding approximate solutions for the linear Fredholm integral equations of the first kind.

\section{References}

[1] A. Adawi, F. Awawdeh, and H. Jaradat, "A numerical method for solving linear integral equations," International Journal of Contemporary Mathematical Sciences, vol. 4, no. 9-12, pp. 485496, 2009.

[2] M. H. AliAbadi and S. Shahmorad, "A matrix formulation of the tau method for Fredholm and Volterra linear integrodifferential equations," The Korean Journal of Computational o Applied Mathematics, vol. 9, no. 2, pp. 497-507, 2002. 
[3] S. Abbasbandy, "Numerical solutions of the integral equations: homotopy perturbation method and Adomian's decomposition method," Applied Mathematics and Computation, vol. 173, no. 1, pp. 493-500, 2006.

[4] F. Mirzaee, "Numerical solution for Volterra integral equations of the first kind via quadrature rule," Applied Mathematical Sciences, vol. 6, no. 17-20, pp. 969-974, 2012.

[5] S. Abbasbandy and E. Babolian, "Automatic augmented Galerkin algorithms for linear first kind integral equations: nonsingular and weak-singular kernels," Bulletin of the Iranian Mathematical Society, vol. 21, no. 1, pp. 35-62, 1995.

[6] V. Marinca and N. Herişanu, "Application of optimal homotopy asymptotic method for solving nonlinear equations arising in heat transfer," International Communications in Heat and Mass Transfer, vol. 35, no. 6, pp. 710-715, 2008.

[7] N. Herişanu, V. Marinca, T. Dordea, and G. Madescu, "A new analytical approach to nonlinear vibration of an electrical machine," Proceedings of the Romanian Academy. Series A, vol. 9, no. 3, pp. 229-236, 2008.

[8] M. S. Hashmi, N. Khan, and S. Iqbal, "Optimal homotopy asymptotic method for solving nonlinear Fredholm integral equations of second kind," Applied Mathematics and Computation, vol. 218, no. 22, pp. 10982-10989, 2012.

[9] A. Sulaiman and I. Hassan, "Successive approximation method (S.A.M.) for solving integral equation of the first kind with symmetric kernel," Journal of Education and Sciences, vol. 21, no. 4, pp. 149-159, 2008.

[10] A. J. Mohammed and J. I. Mustafa, "Construction of a new technique in Aitken extrapolation method for solving Fredholm integral equation of the first kind with iterated kernel," Journal of Education and Sciences, vol. 21, no. 2, pp. 143-149, 2008. 


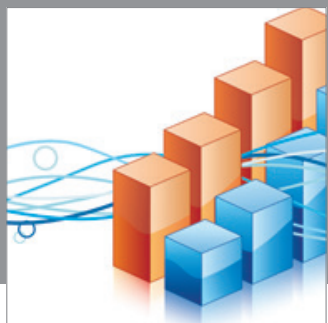

Advances in

Operations Research

mansans

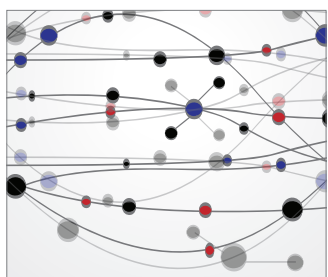

The Scientific World Journal
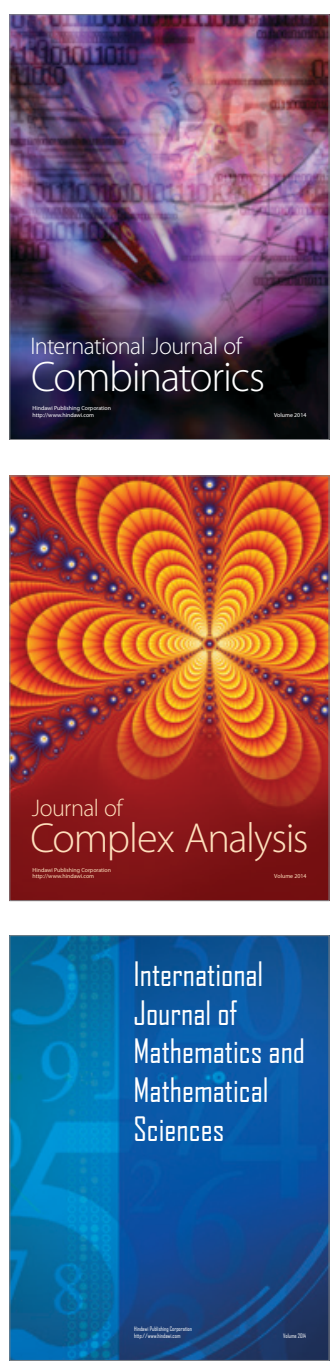
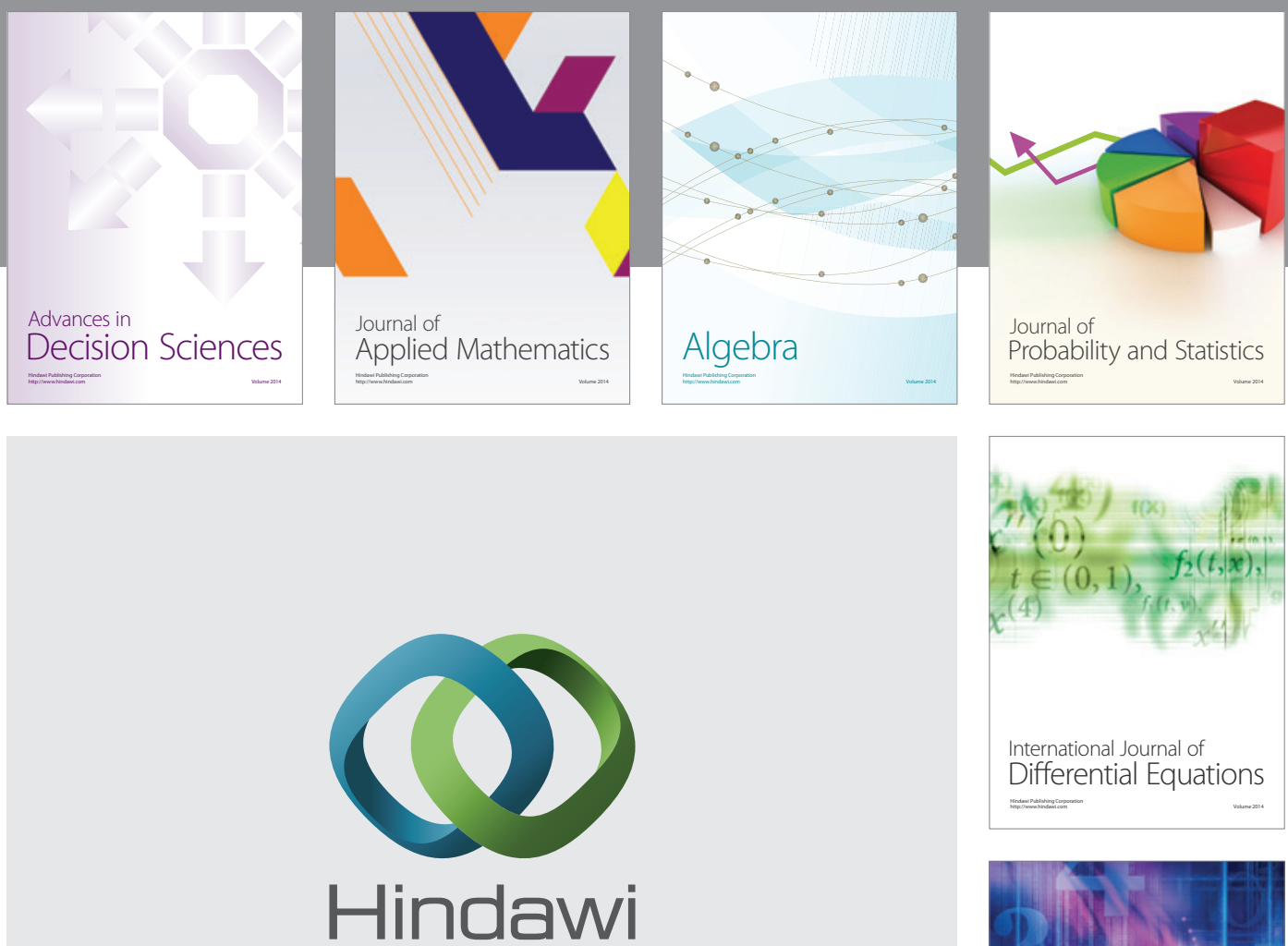

Submit your manuscripts at http://www.hindawi.com
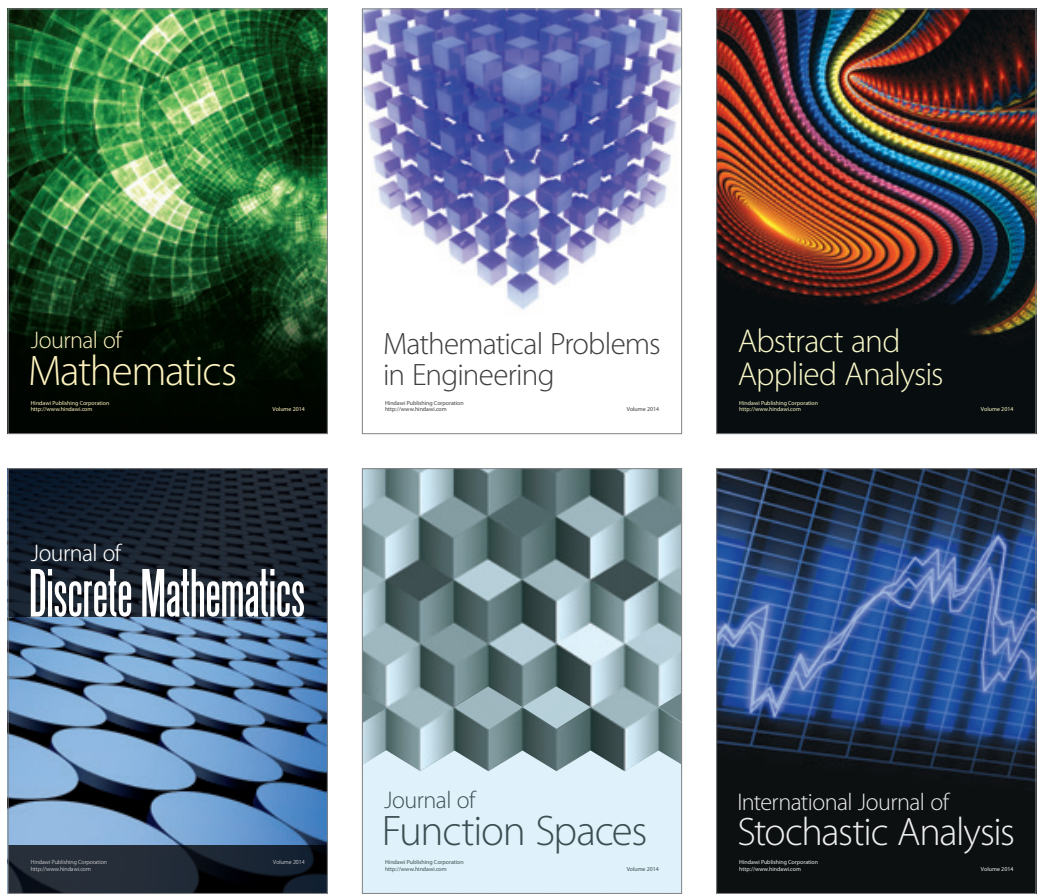

Journal of

Function Spaces

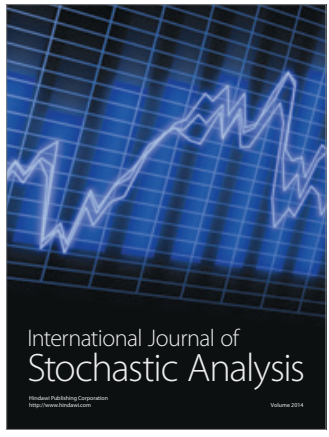

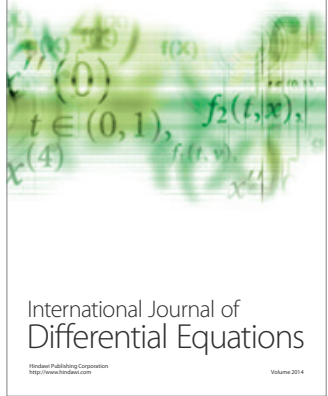
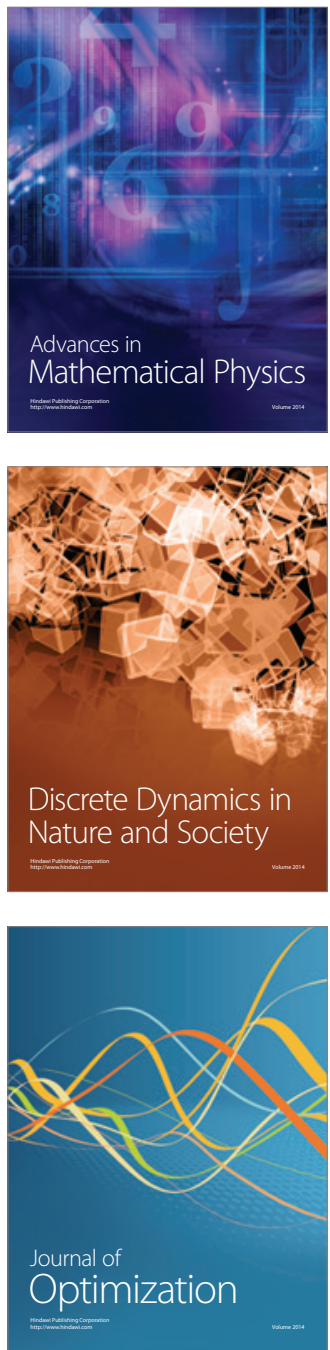